\title{
Contrast-enhanced ultrasound with sulphur- hexafluoride in diagnosis of early HCC in cirrhosis
}

\author{
Antonio Giorgio', Pietro Gatti ${ }^{2}$, Paolo Matteucci ${ }^{3}$, Valentina Giorgio ${ }^{4}$ \\ 'Department of Medicine, Interventional Ultrasound Unit, Athena Clinical Institute, Caserta 81016, Italy. \\ ${ }^{2}$ Department of Medicine, Brindisi General Hospital, Brindisi 72100, Italy. \\ ${ }^{3}$ Radiation Oncology Institute, Campus Biomedico University, Rome 00128, Italy. \\ ${ }^{4}$ Fondazione Policlinico A.Gemelli IRCCS, Department of Woman and Child Health and Public Health, Roma 00168, Italy.
}

Correspondence to: Dr. Antonio Giorgio, Department of Medicine, Interventional Ultrasound Unit, Athena Clinical Institute, Caserta, Italy. E-mail: agiorgio28@gmail.com

How to cite this article: Giorgio A, Gatti P, Matteucci P, Giorgio V. Contrast-enhanced ultrasound with sulphur-hexafluoride in diagnosis of early HCC in cirrhosis. Hepatoma Res 2019;5:20. http://dx.doi.org/10.20517/2394-5079.2019.05

Received: 12 Jan 2019 First Decision: 25 Mar 2019 Revised: 29 Apr 2019 Accepted: 7 May 2019 Published: 13 Jun 2019

Science Editor: Jin-Lin Hou Copy Editor: Cai-Hong Wang Production Editor: Jing Yu

\begin{abstract}
Contrast-enhanced ultrasound (CEUS) with pure blood stream contrast agents allow the study of blood supply of focal liver lesions and especially of hepatocellular carcinoma (HCC) in cirrhosis. Its sensitivity and specificity in diagnosis of small tumors is very high. This review summarizes the recent results on CEUS with SonoVue, which is one of the second generation contrast agents, in the diagnosis of early HCC in cirrhosis emphasizing its increasing role in routine clinical practice.
\end{abstract}

Keywords: Hepatocellular carcinoma, contrast-enhanced ultrasound, cirrhosis, early hepatocellular carcinoma

\section{INTRODUCTION}

Today the use of dynamic imaging modalities allows the study of liver vasculature and in particular the study of the blood supply of the focal liver lesions. Focal liver lesions can be non-invasively differentiated and characterized in benign or malignant on the basis of their vascular support, so to avoid the need of biopsy. This revolution was provided non-invasively by the dynamic study of liver vasculature using contrastenhanced CT (CECT), contrast-enhanced MRI (CEMRI) and, lastly, Contrast-enhanced ultrasound (CEUS). These new dynamic imaging techniques have had a strong impact especially in the field of diagnosis of hepatocellular carcinoma (HCC) in cirrhosis, and have achieved considerable importance in the management

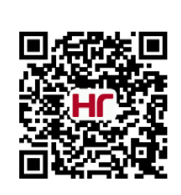


of this kind of tumor. HCC is the most frequent primary epitelial malignant tumor of the liver ${ }^{[1]}$. The main feature of this tumor is that it arises mainly in patients with cirrhosis trough the transformation of normal regenerating nodules in displastic nodules and finally overt $\mathrm{HCC}^{[1-5]}$. For non cirrhosis HBV patients, it is via HBV-DNA integration into the host genome, which occurs at early steps of clonal tumor expansion and induces both genomic instability and direct insertional mutanegesis. Therefore, patients with cirrhosis represent a high risk population for developing HCC and should undergo a 6 months surveillance with ultrasound (US) to allow the detection of the tumor at an early stage ${ }^{[6-13]}$. In clinical practice, CECT and CEMRI are not recommended in the surveillance programs. Viceversa, when US examination shows a new nodule, CECT and/or CEMRI are reccomended for the staging of the disease.

\section{VASCULAR CHANGES IN HEPATOCARCINOGENESIS AND CEUS}

It is well known that in the case of HCC arising on cirrhosis the normal vascular support is reversed: while in normal subjects the vascular support of the liver is provided by the portal venous system up to $75 \%$, in HCC the blood supply of the nodule is only arterial.

The process of hepatocarcinogenesis in cirrhosis includes the progression from Low Grade Displastic nodule to High Grade Displastic nodule (HGDN) and overt HCC. During this process, unpaired arteries progressively substitute tumoral portal tracts so that overt HCC blood supply is only arterial. This pathological phenomenon explains the arterial hyperenhancement of typical HCC nodules on dynamic imaging modalities such as CECT, CEMRI and CEUS ${ }^{[1,15]}$.

In recent years, CEUS has gained significant popularity in the characterization of focal liver lesions. CEUS has shown to have a great capability in distinguishing between benign or malignant hepatic nodules on the basis of characteristic patterns of blood supply of the lesions ${ }^{[16]}$. The 2nd generation contrast agent SonoVue is a pure blood stream agent formed by micro bubbles with inert gas sulphur- hexafluoride and a palmitic acid shell. After Intra venam injection of $2.4 \mathrm{~mL}$ of SonoVue, in real time and second by second, the arterial phase appearance of contrast agent distribution within the nodule's vessels (duration 10-30 s after contrast injection) can be studied and recorded, followed by the portal phase (30-60 s after injection) and the late or sinusoidal phase (60-240 s) ${ }^{[16,17]}$. The typical CEUS pattern of HCC in cirrhosis is reported in Figures $1-5^{[18,19]}$.

\section{HCC DIAGNOSIS IN CIRRHOSIS AND ROLE OF CEUS US surveillance of HCC in cirrhosis and CEUS}

It is well known that conventional US, although a unique tool for surveillance, has a great sensitivity but a very low specificity in the characterization of HCC in cirrhosis ${ }^{[16]}$. CEUS has shown to significantly improve US accuracy ${ }^{[16,20]}$. CEUS using SonoVue easily show the characteristics of liver nodules blood supply and therefore allow the characterization of malignant nodules ${ }^{[17]}$.

CEUS has determined a real revolution by eliminating the low specificity of conventional US in diagnosing and managing HCC after recognition of a new nodule in a cirrhotic liver: this is due to the immediate and real time visualization of its vascular supply ${ }^{[16]}$. Other advantages of CEUS are the absence of ionizing radiation, the low cost, repeatability, safety and, more important, the possibility to be performed in patients with renal insufficiency ${ }^{[1,221]}$. Moreover, it has been reported that CEUS add significant diagnostic information in the characterization of atypical or indistinctive lesions on conventional US ${ }^{[21,22]}$.

The main limitation of CEUS using pure blood stream contrast agents is based on the fact that only one lesion at a time can be studied and characterized, due to the very short duration of the arterial phase (see later). Consequently, CECT and/or CEMRI are the only dynamic imaging modalities to be used for the staging of the tumor. For the same reasons, CEUS with pure blood stream agents such as sulphur hexafluoride cannot be used for surveillance, unlike Sonazoid which is a new US contrast agent using perflubutane (see later). 


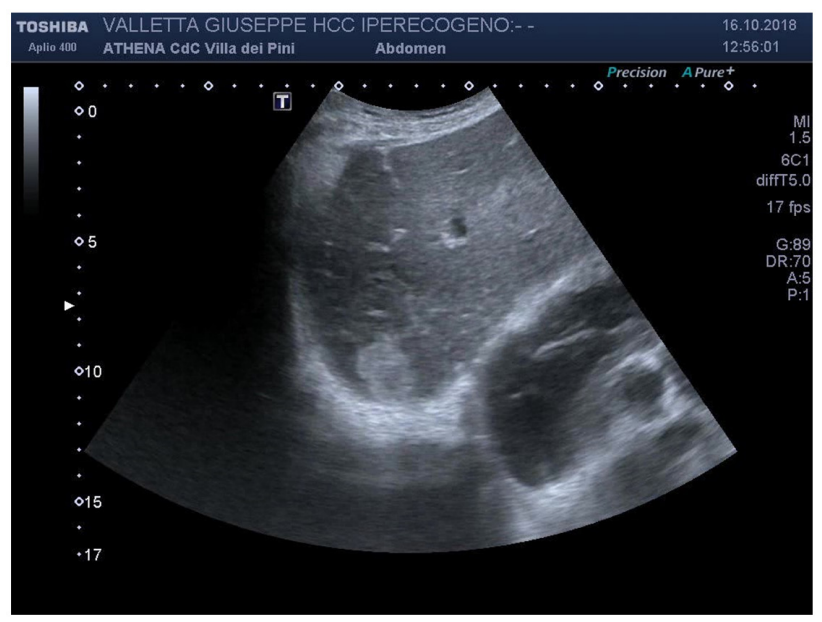

Figure 1. A 75-year old man with HCV related cirrhosis, successfully treated one year before with DAAs and presenting with a small $2 \mathrm{~cm}$ angioma-like hyperchoic nodule in the 7th segment of the liver, not present during the surveillance in the previous ultrasound (US) exam 6 months before

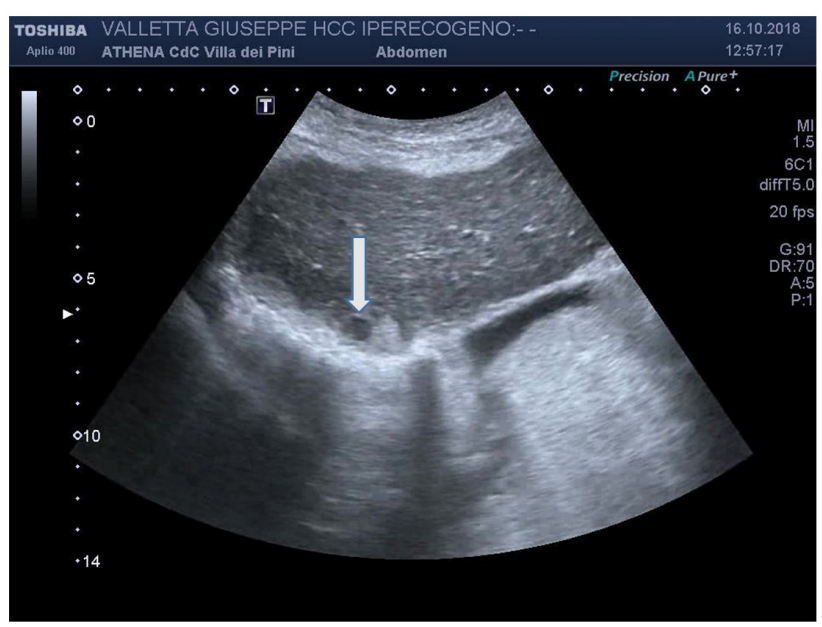

Figure 2. A very small hypoechoic round portion (white arrow) is seen within the hyperechoic nodule ( nodule in nodule)

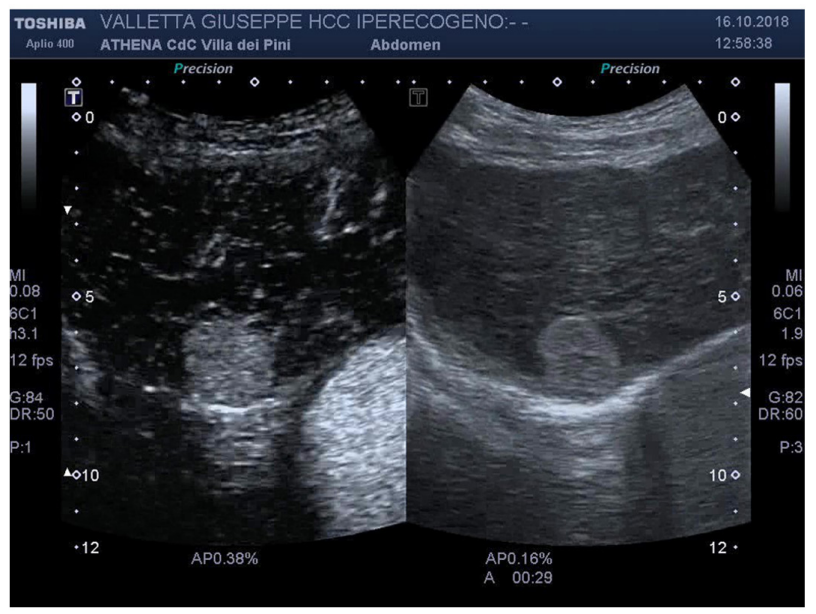

Figure 3. CEUS appearence: in the arterial phase, the nodule becomes homogeneously hyperechoic (hyperehancenced) (left of the figure) 


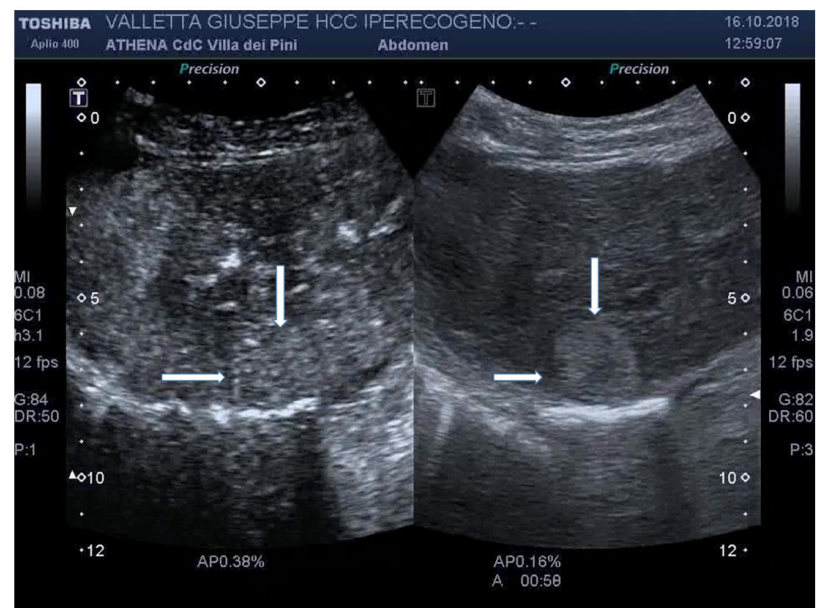

Figure 4. At the end of the portal phase (59 s) the nodule (white arrows) appears iso-enhanced

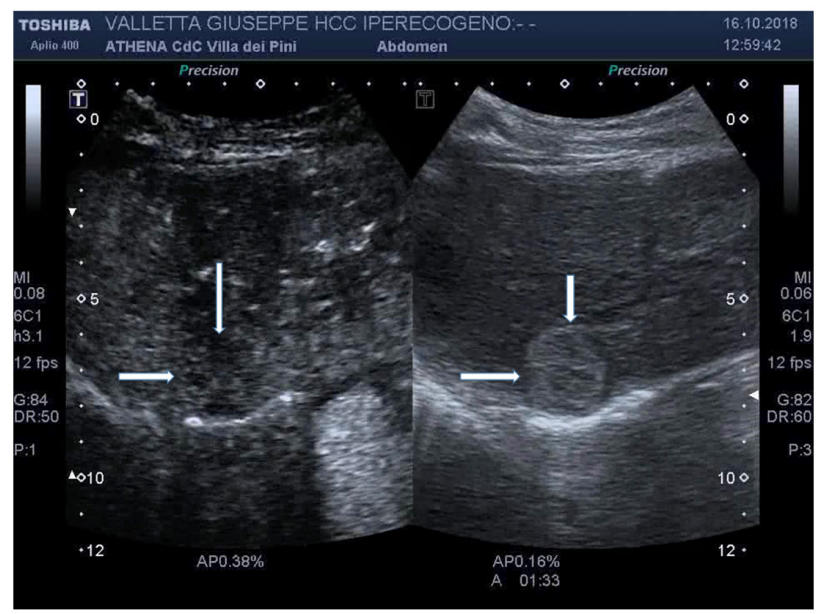

Figure 5. Only at $1.33 \mathrm{~min}$ in the late phase, the nodule (white arrows) becomes slightly hypoechic (hypoenhanced)

\section{CEUS patterns of small HCC in cirrhosis and international guidelines}

Nowadays, all practice guidelines on the management of HCC in cirrhosis have endorsed CEUS as a dynamic imaging modality capable of diagnosing HCC in cirrhosis per lesion ${ }^{[8-13]}$. The Italian Society for the Study of the Liver, in cooperation with the Italian Societies of Oncology, Radiology, Surgery, Hepatobiliary Surgery and Organs Transplant, published a position paper stating that CEUS can diagnose non-invasively as HCC a hepatic nodule when the characteristics of arterial hyperenhancement and wash out are present, like CECT and CEMRI ${ }^{[12]}$. The guidelines of CEUS hepatic applications of EFSUMB and WFUMB date back to $2012^{[13]}$.

The recent EASL guidelines for the management of HCC recognized that "CEUS can be effectively utilized to characterise lesions in cirrhosis" although CT and MRI are panoramic technique useful for the staging of the tumor, since the rapid CEUS arterial enhancement does not allow the detection of eventual multiple nodules scattered in the liver ${ }^{[10]}$. In fact, CEUS "can be utilised to characterise one or few nodules detected on conventional US surveillance". Nonetheless, EASL does not recommend CEUS as first line tool, but only in cases where CT/MRI are contraindicated or inconclusive ${ }^{[10]}$.

2017 Chinese guidelines stated that in HBV/HCV chronic hepatitis or cirrhosis patients diagnosis of HCC > $2 \mathrm{~cm}$ can be made with only one dynamic imaging tool (CECT, CEMRI or CEUS) when the typical findings are present. If the nodule has a diameter $<2 \mathrm{~cm}$, two dynamic imaging techniques are needed ${ }^{[23]}$. 
The new 2017 Japanese guidelines on management of HCC stated that CEUS sensitivity is similar to dynamic CT or dynamic MRI in diagnosis of HCC and therefore CEUS is able to characterize nodules detected on sonography. The US contrast agent indicated in these guidelines is Sonazoid, which is a US contrast agent phagocytized by Kupffer cells ${ }^{[24]}$. In the past, controversies arose over the possibility of misdiagnosis between small $(<3 \mathrm{~cm})$ Intrahepatic Cholangiocarcinoma (ICC) arising in cirrhosis and HCC. In such cases, CEUS was considered unable to distinguish between these two entities in cirrhotic livers because, in the experience of Spanish authors, CEUS washout patterns of ICC can mimic those of $\mathrm{HCC}^{[25]}$. As of today, it is well established that the differential diagnosis between small $<3 \mathrm{~cm} \mathrm{ICC}$ in cirrhosis and small HCC is no longer a problem. It is well known that, at CEUS, small ICC can present an intense (as HCC) arterial phase hyperenhancement, but a more rapid and marked washout in the portal phase (always $<42 \mathrm{~s}$ ), differently from the mild and very late wash-out (> $60 \mathrm{~s})$ of HCC in the sinusoidal phase, avoiding any pitfalls $\mathrm{s}^{[26,27]}$. Therefore, the old diatribe that for several years has labelled CEUS not able to distinguish between small HCC and ICC nodules arisen in a cirrhotic liver is now to be considered surpassed ${ }^{[2-31]}$. Nevertheless, we should consider that small HCC nodules $(<2 \mathrm{~cm})$ can present with hyperenhancement in the arterial phase followed by isovascularity in the portal and sinusoidal phases in more than $50 \%$ of cases (as is shown in Figure 4 and Giorgio's 2011 results .as reported below.

\section{CEUS LI-RADS and HCC}

A so-called CEUS LI-RADS was proposed by the American College of Radiology based on the Liver Imaging Reporting and Data System (LI-RADS) using CECT and CEMRI patterns for HCC in cirrhotic livers. LIRADS was originally developed for CECT and CEMRI, but expanded to include CEUS. Based on CEUS features, focal liver lesions ("observations" in radiologic terminology) detected in a cirrhotic liver can be classified in 5 major classes ranging from "definitely benign" (LR-1) to "definitely HCC" (LR-5) ${ }^{[32]}$. Sonovue is included in the CEUS LI- RADS version 2017 $7^{[32]}$. The 5 major categories (LR-1-LR-5) are classified according to the diameter of the lesions and their contrast enhancement patterns.

The CEUS pattern characterized by the presence of rapid, intense and homogeneous hyperenhancement in the arterial phase (APHE) followed by mild and late ( $>60 \mathrm{~s}$ ) wash-out is termed as CEUS LI-R 5 . When a hepatic nodule discovered in a cirrhotic liver presents with the CEUS LR-5 pattern, the nodule can managed as HCC and there is no need for biopsy. This classification is applied to nodules $>10 \mathrm{~mm}^{[32-35]}$.

Very recently, Terzi et al. ${ }^{[36]}$ reported very interesting data that strongly influenced the last 2018 EASL guidelines in diagnosis of HCC. In a multicentre retrospective study, these authors evaluated CEUS patterns of 1,006 nodules in 848 patients with chronic liver disease at risk for HCC. Median size of nodules was small: $2 \mathrm{~cm}$. Five hundred twenty one (52\%) out of all nodules showed APHE and a mild, late wash-out. The $17 \%$ of nodules showed APHE and isoechogenicity in the portal and late phase, while $16 \%$ of nodules were iso-enhancing in the arterial and portal-late phases. The most important data was that 512 (98.5\%) of all nodules classified as CEUS LR-5 were HCC. When authors included in their analysis 3 other CEUS LR-5 cases that were judged underdiagnosed and that resulted HGDN at biopsy, the rate of HCC diagnosis became 99\%. In their study, Terzi et al. ${ }^{[36]}$ did not report any case of misdiagnosis with ICC.

Moreover, studies on inter-observer agreement suggest that the classification of small hepatic nodules $(<2 \mathrm{~cm})$ with LI-RADS-CEUS is reproducible with good consistency in patients with chronic liver disease ${ }^{[37-39]}$.

\section{CEUS arterial hyper enhancement and early HCC}

Some authors studied the interobserver agreement for CEUS-based standardized algorithms in diagnosis of HCC in high-risk patients. The interobserver agreement was good for arterial phase hyper enhancement, which is the key diagnostic feature for HCC nodules in a cirrhotic liver ${ }^{[39]}$. For what has been said so far, although it is evident that HCC diagnosis on CEUS relies also on the washout findings (type and time), 
arterial hyper-enhancement remains the main element for the visualization of the HCC nodule when pure blood stream contrast agents are used.

This feature was also studied by Giorgio et al. ${ }^{[40]}$ who reported a considerable effectiveness of CEUS in detection of arterial hyperenhancement in small nodules $(7-20 \mathrm{~mm})$ discovered in cirrhotic patients during surveillance, so to shorten the diagnostic work-up for the management of HCC.

In Giorgio's experience, CEUS showed arterial hyperenhancement in 95.5\% of HCC nodules, with a sensitivity of $94.48 \%$, a specificity of $100 \%$ and $100 \%$ PPV. In this study, CEMRI showed $97 \%$ sensitivity, $80 \%$ specificity and $97 \%$ PPV. The authors concluded that CEUS has a great capability in detection of arterial hypervascularity in $<2 \mathrm{~cm}$ HCC. In Giorgio et al. ${ }^{[40]}$ 's experience, only $4.5 \%$ of new nodules escaped the demonstration of arterial hyervascularity. Therefore authors concluded that "CEUS must be performed immediately after conventional US to contrast the malignant fate of small lesions arising in a cirrhotic liver". Moreover, "CEUS should be included in the diagnostic management of HCC in order to avoid a late diagnosis, enable an early treatment and improve survival".

It was shown that CEUS vascular patterns of HCC lesions are related to size and histologic differentiation of the tumor. Ling et al.$^{[4]]}$ reported that $<3 \mathrm{~cm}$ HCC nodules show more homogeneous hyperenhancement compared to $>3 \mathrm{~cm}$ lesions ${ }^{[41]}$. Moreover, heterogeneous arterial enhancement of $\mathrm{HCC}$ nodules $>3 \mathrm{~cm}$ were followed by faster washout compared to $<3 \mathrm{~cm}$ nodules. The portal and late phase washout was faster in poorly differentiated HCC compared to well-differentiated lesions.

Italian authors also reported that CEUS has high capability in the differential diagnosis of dysplastic nodules (DN), early hepatocellular carcinoma and progressed $\mathrm{HCC}^{[42]}$. According to this study, DN, early HCC and progressed HCC have different and characteristic CEUS patterns. Progressed HCC is characterized by rapid, intense and homogeneous arterial hyperenhancement, while early HCC displays the so called "reticular pattern". This pattern is characterized by inhomogeneous enhancement during arterial phase and complete enhancement in the late phase. In the experience of the authors, the "reticular pattern" identified early HCC nodules with a sensitivity of $85.7 \%$ and a specificity of $96.1 \%^{[42]}$.

\section{Comparison among CEUS, enhanced CT and enhanced MRI in diagnosis of small $(2-3 \mathrm{~cm}) \mathrm{HCC}$ nodules in cirrhosis}

Many authors studied the diagnostic capability of CECT, CEMRI and CEUS alone or in combination for the diagnosis of small HCC on cirrhosis. Aubé et al. ${ }^{[43]}$ carried out a large multicentre study in a large number of cirrhotic patients (544 nodules in 381 patients). Authors aimed at evaluating the accuracy of CECT, CEMRI and CEUS alone and in combination, in diagnosing small $(10-30 \mathrm{~mm})$ HCC nodules. The best combination for the 10-20 mm nodules was CEMRI -CECT. They found that, when a first imaging tool was inconclusive and CEUS was used as second dynamic technique, this combination allowed the highest specificity with only a slight drop of sensitivity for 10-20 mm nodules and the highest sensitivity and specificity for $20-30 \mathrm{~mm}$ nodules. The authors concluded that in diagnosis of small HCC nodules the best combination is CEMRI followed by CEUS ${ }^{[43]}$.

Moudgil et al.$^{[44]}$ compared the role of CEUS and CECT in diagnosis of HCC. In their experience, CEUS and CECT were similar in demonstrating the arterial hypervascularity of HCC nodules. Vice versa, they found a better capability of CEUS in the demonstration of washout pattern and the presence of the capsule of the nodules, when present .

Finally, Intraoperative Contrast-Enhanced Ultrasound (CEUS/IOCEUS) is routinely performed during surgical resection of HCC in cirrhosis. It has been shown that such technique allows the detection of 
additional liver lesions. This advantage was demonstrated when IOCEUS was compared to preoperative MRI, as well as to preoperative CEUS. According to results of Huf et al ${ }^{[45]}$, in $27 \%$ of their cases IOCEUS allowed the detection of further liver lesions not detected preoperatively. Such detection of further lesions modified the treatment planning and resection was extended if necessary.

\section{CONCLUSION}

Today, CEUS plays an essential role in the clinical recognition of small nodules arising de novo or recurrent in cirrhotic livers at risk for HCC. The advantages of CEUS over CT/MRI are unique and are represented by: the high sensitivity in depiction of arterial hypervascularity of HCC; the better demonstration of rapid washout for non-HCC malignant nodules; the very late washout of HCC.

In 2016, SonoVue was approved for the first time in the United States for the diagnostic imaging of liver tumors in adults and children. It is undisputable that this approval represents a milestone for CEUS ${ }^{[46]}$. In clinical practice, CEUS demands are constantly increasing in Europe, Asia and Canada (and we are hoping also in USA after the FDA approval) in the Hepatology Units and not only (see Gastroenteroly Units, Infectious disease Units, Internal Medicine Units and Surgical Units).

It is undoubtable that the most important benefit of CEUS is based on the fact that physicians can perform CEUS soon after the detection on conventional US of a new nodule during surveillance of cirrhotic patients. Thanks to this technique, physicians can immediately exclude typical benignancy, non-HCC malignant nodules such as ICC and, mainly, in case of CEUS recognition of early HCC, physicians can define a rapid therapeutic work-up choosing among liver transplantation, resection or ablation.

\section{DECLARATIONS}

\section{Authors' contributions}

Design of the work, data analysis and interpretation: Giorgio A

Data acquisition, material support: Giorgio A, Gatti P, Matteucci P, Giorgio V

Wrote the manuscript: Giorgio A, Giorgio V

\section{Availability of data and materials}

Not applicable.

\section{Financial support and sponsorship}

None.

\section{Conflicts of interest}

All authors declared that there are no conflicts of interest.

\section{Ethical approval and consent to participate}

Not applicable.

\section{Consent for publication}

Not applicable.

\section{Copyright}

(c) The Author(s) 2019.

\section{REFERENCE}

1. Van Malenstein H, van Pelt J, Verslype C. Molecular classification of hepatocellular carcinoma anno 2011. Eur J Cancer 2011;47:1789-97. 
2. El-Serag HB, Davila JA, Petersen NJ, McGlynn KA. The continuing increase in the incidence of hepatocellular carcinoma in the United States: an update. Ann Intern Med 2003;139:817-23.

3. McGlynn KA, London WT. Epidemiology and natural history of hepatocellular carcinoma. Best Pract Res Clin Gastroenterol 2005;19:3-23.

4. Trevisani F, Cantarini MC, Wands JR, Bernardi M. Recent advances in the natural history of hepatocellular carcinoma. Carcinogenesis 2008;29:1299-305.

5. Ho DW, Lo RC, Chan LK, Ng IO. Molecular pathogenesis of hepatocellular carcinoma. Liver Cancer 2016;5:290-302.

6. Poon RT, Fan ST, Lo CM, Liu CL, Wong J. Long-term survival and pattern of recurrence after resection of small hepatocellular carcinoma in patients with preserved liver function: implications for a strategy of salvage transplantation. Ann Surg 2002;235:373-82.

7. Arii S, Yamaoka Y, Futagawa S, Inoue K, Kobayashi Kojiro M, et al. Results of surgical and nonsurgical treatment for small-sized hepatocellular carcinomas: a retrospective and nationwide survey in Japan. The Liver Cancer Study Group of Japan. Hepatology 2000;32:1224-9.

8. Omata M, Cheng AL, Kokudo N, Kudo M, Lee JM, et al. Asia-Pacific clinical practice guidelines on the management of hepatocellular carcinoma: a 2017 update. Hepatol Int 2017;11:317-70.

9. Korean Liver Cancer Study Group (KLCSG) and National Cancer Center, Korea (NCC). 2014 KLCSG-NCC Korea practice guideline for the management of hepatocellular carcinoma. Gut Liver 2015;9:267-317.

10. Julie K. Heimbach, Laura M. Kulik, Richard S. Finn,Claud. AASLD guidelines for the treatment of hepatocellular carcinoma. Hepatology 2018;67:358-80.

11. European Association for the Study of the Liver. EASL clinical practice guidelines: management of hepatocellular carcinoma. J Hepatol 2018;69:182-236.

12. Italian Multi-Society reccomandations for the integrated management of Hepatocelluar Carcinoma. Published on line dec 22, 2106. Available from: https://www.sirm.org/wp-content/uploads/2019/03/HCC.pdf. [Last accessed on 24 May 2019]

13. Claudon M, Dietrich CF, Choi BI, Cosgrove DO, Kudo M, et al. Guidelines and good clinical practice recommendations for contrast enhanced ultrasound (CEUS) in the liver - update 2012: a WFUMB-EFSUMB initiative in cooperation with representatives of AFSUMB, AIUM, ASUM, FLAUS and ICUS. Ultrasound Med Biol 2013;39:187-210.

14. Choi JY, Lee JM, Sirlin CB. CT and MR imaging diagnosis and staging of hepatocellular carcinoma: part I. Development, growth, and spread: key pathologic and imaging aspects. Radiology 2014;272:635-54.

15. International Consensus Group for Hepatocellular Neoplasia. Pathologic diagnosis of early hepatocellular carcinoma: a report of theinternational consensus group for hepatocellular neoplasia. Hepatology2009;49:658-64.

16. Quaia E, Calliada F, Bertolotto M, Rossi S, Garioni L, et al. Characterization of focal liver lesions with contrast-specific US modes and a sulfur hexafluoride-filled microbubble contrast agent: diagnostic performance and confidence. Radiology 2004;232:420-30.

17. Salvatore V, Gianstefani A, Negrini G, Allegretti G, Galassi M, et al. Imaging diagnosis of hepatocellular carcinoma: recent advances of contrast-enhanced ultrasonography with SonoVue®. Liver Cancer 2016;5:55-66.

18. Giorgio A, Ferraioli G, Tarantino L, de Stefano G, Scala V, et al. Contrast-enhanced sonographic appearance of hepatocellular carcinoma in patients with cirrhosis: comparison with contrast-enhanced helical CT appearance. AJR Am J Roentgenol 2004;183:1319-26.

19. Giorgio A, De Stefano G, Coppola C, Ferraioli G, Esposito V, et al. Contrast-enhanced sonography in the characterization of small hepatocellular carcinomas in cirrhotic patients: comparison with contrast-enhanced ultrafast magnetic resonance imaging. Anticancer Res 2007;27:4263-9.

20. Quaia E, Lorusso A, Grisi G, Stacul F, Cova MA. The role of CEUS in the characterization of hepatocellular nodules detected during the US surveillance program--current practices in Europe. Ultraschall Med 2012;33 Suppl 1:S48-56.

21. Dong Y, Mao F, Cao J, Fan P, Wang WP. Characterization of focal liver lesions indistinctive on b mode ultrasound: benefits of contrastenhanced ultrasound. Biomed Res Int 2017;2017:8970156.

22. Dong Y, Wang WP, Mao F, Dietrich C. Contrast-enhanced ultrasound features of hepatocellular carcinoma not detected during the screening procedure. Z Gastroenterol 2017;55:748-53.

23. Xie DY, Ren ZG, Zhou J, Fan J, Gao Q. Critical appraisal of Chinese 2017 guideline on the management of hepatocellular carcinoma. Hepatobiliary Surg Nutr 2017;6:387-96.

24. Kudo M, Matsui O, Izumi N, Iijima HD, Kadoya M, et al. JSH consensus-based clinical practice guidelines for the management of hepatocellular carcinoma: 2014 update by the liver cancer study group of Japan. Liver Cancer 2014;3:458-68.

25. Vilana R, Forner A, Bianchi L, García-Criado A, Rimola J, et al. Intrahepatic peripheral cholangiocarcinoma in cirrhosis patients may display a vascular pattern similar to hepatocellular carcinoma on contrast-enhanced ultrasound. Hepatology 2010;51:2020-9.

26. Wildner D, Bernatik T, Greis C, Seitz K, Neurath MF, et al. CEUS in hepatocellular carcinoma and intrahepatic cholangiocellular carcinoma in 320 patients - early or late washout matters: a subanalysis of the DEGUM multicenter trial. Ultraschall Med 2015;36:132-9.

27. de Sio I, Iadevaia MD, Vitale LM, Niosi M, Del Prete A, et al. Optimized contrast-enhanced ultrasonography for characterization of focal liver lesions in cirrhosis: a single-center retrospective study. United European Gastroenterol J 2014;2:279-87.

28. Giorgio A, Calisti G, Giorgio V. CEUS and HCC: are the 2008 EFSUMB guidelines still valid or has their wash-out already started? Ultraschall Med 2011;32:315-6.

29. Giorgio A, Montesarchio L, Giorgio V. Misdiagnosis of intrahepatic cholangiocarcinoma in cirrhosis at contrast-enhanced ultrasound: a real new clinical entity or just an exaggerated fear? Liver Int 2013;33:1608.

30. Giorgio A. Diagnostic algorithm of hepatocellular carcinoma on cirrhosis: CEUS or no CEUS, that is the problem. Dig Liver Dis 2011;43:499. 
31. Jang HJ, Kim TK, Burns PN, Wilson SR. CEUS: an essential component in a multimodality approach to small nodules in patients at high-risk for hepatocellular carcinoma Eur J Radiol 2015;84:1623-35.

32. Kono Y, Lyshchik A, Cosgrove D, Dietrich CF, Jang HJ, et al. Contrast enhanced ultrasound (CEUS) liver imaging reporting and data system (LI-RADS(R)): the official version by the american college of radiology (ACR). Ultraschall Med 2017;38:85-6.

33. Piscaglia F, Wilson SR, Lyshchik A, Cosgrove D, Dietrich CF, et al. American college of radiology contrast enhanced ultrasound liver imaging reporting and data system (CEUS LI-RADS) for the diagnosis of hepatocellular carcinoma: a pictorial essay. Ultraschall Med 2017;38:320-4.

34. Tae Kyoung Kim, Seung Yeon Noh, Stephanie R Wilson, Yuko Kono, Fabio Piscaglia, et al. Contrast-enhanced ultrasound (CEUS) liver imaging reporting and data system (LI-RADS) 2017 - a review of important differences compared to the CT/MRI system. Clin Mol Hepatol 2017;23:280-9.

35. Dietrich CF, Potthoff A, Helmberger T, Ignee A, Willmann JK, et al. Contrast-enhanced ultrasound: liver imaging reporting and data system (CEUS LI-RADS)]. Z Gastroenterol 2018;56:499-506. (in German)

36. Terzi E, Iavarone M, Pompili M, Veronese L, Cabibbo G, et al. Contrast ultrasound LI-RADS LR-5 identifies hepatocellular carcinoma in cirrhosis in a multicenter restropective study of 1,006 nodules; CEUS LI-RADS Italy study group collaborators. J Hepatol 2018;68:48592.

37. Yang D, Li R, Tang CL, Ma KS, Guo DY, et al. Perfusion characteristics of hepatocellular carcinoma at contrast-enhanced ultrasound: influence of the cellular differentiation, the tumor size and the underlying hepatic condition. Sci Rep 2018;8:4713.

38. Schellhaas B, Hammon M, Strobel D, Pfeifer L, Kielisch C, et al. Interobserver and intermodality agreement of standardized algorithms for non-invasive diagnosis of hepatocellular carcinoma in high-risk patients: CEUS-LI-RADS versus MRI-LI-RADS. Eur Radiol 2018;28:4254-64.

39. Schellhaas B, Pfeifer L, Kielisch C, Goertz RS, Neurath MF, et al. Interobserver agreement for contrast-enhanced ultrasound (CEUS)based standardized algorithms for the diagnosis of hepatocellular carcinoma in high-risk patients. Ultraschall Med 2018;39:667-74.

40. Giorgio A, Montesarchio L, Gatti P, Amendola F, Matteucci P, et al. Contrast-enhanced ultrasound: a simple and effective tool in defining a rapid diagnostic work-up for small nodules detected in cirrhotic patients during surveillance. J Gastrointestin Liver Dis 2016;25:205-11.

41. Ling W, Wang M, Ma X, Qiu T, Li J, et al. The preliminary application of liver imaging reporting and data system (LI-RADS) with contrast-enhanced ultrasound (CEUS) on small hepatic nodules ( $\leq 2 \mathrm{~cm})$. J Cancer 2018;9:2946-52.

42. Giorgio A, Calisti G, di Sarno A, Farella N, de Stefano G, et al. Characterization of dysplastic nodules, early hepatocellular carcinoma and progressed hepatocellular carcinoma in cirrhosis with contrast-enhanced ultrasound. Anticancer Res 2011;31:3977-82.

43. Aubé C, Oberti F, Lonjon J, Pageaux G, Seror O, et al. EASL and AASLD recommendations for the diagnosis of HCC to the test of daily practice. Liver Int 2017;37:1515-25.

44. Moudgil S, Kalra N, Prabhakar N, Dhiman RK, Behera A, et al. Comparison of contrast enhanced ultrasound with contrast enhanced computed tomography for the diagnosis of hepatocellular carcinoma. J Clin Exp Hepatol 2017;7:222-9.

45. Huf S, Platz Batista da Silva N, Wiesinger I, Hornung M, Scherer MN, et al. Analysis of liver tumors using preoperative and intraoperative contrast-enhanced ultrasound (CEUS/IOCEUS) by radiologists in comparison to magnetic resonance imaging and histopathology. Rofo 2017;189:431-40.

46. Seitz K, Strobel D. A milestone: approval of CEUS for diagnostic liver imaging in adults and children in the USA. Ultraschall Med 2016;37:229-32. 